\title{
Die Herkunft des Untergrundes im Kohlebogen-spektrum
}

\author{
R. L. Roes und J. A. Surt \\ Phy'sisch Laboratorium der Rijks.Universiteit, Utrecht
}

\begin{abstract}
In the arc method of spectrochemical analysis the sensitivity, i.e. the measurability of low contents, is limited by the background in the spectrum. For the spectrum lines to be used will disappear into the background when they become weak. With the carbon are in air the disturbing influence is strengthened by the fact that the background is not smooth but contains a large number of weak bands. It is indeed possible to subtract the background from a measured spectral line; however, the correct (graphical) procedure consumes much time, and naturally the accuracy is limited. So a weakening of the background will often be useful, provided that the favourable properties of the arc are not clamaged. Research in this field will be the more effective the more completely the origins of the components of the background are known.

In spectrochemical analysis the spectral background often consists chiefly of the spectrum of the arc itself (without a sample). We have investigated the ultra-violet part of it. The spectrum of the carbon arc in air proved to be readily reproducible and of fairly constant intensity. It contains band structure throughout; apparently the contribution of glowing carbon particles to the arc light is small. Some weak intensity maxima in the near ultra-violet were identified as continuation of the violet $\mathrm{CN}$ bands; they are subject to the same strong influence of the arc current and they are absent when $\mathrm{CO}_{2}$ is used instead of air. Further, the $\mathrm{N}_{2}$ bands 3371 and $3159 \AA$ appear, with their rotational structure; they are more distinct in an arc in $\mathrm{N}_{2}$ than in air, and are absent in $\mathrm{CO}_{2}$. With the free burning arc in air also the $\mathrm{OH}$ bands $3064 \AA$ etc. were noticed, in the emission of the outer zone. In the farther ult ra-violet the structure largely agrees with the bands of $\mathrm{NO}$; these maxima ranish when the air is replaced by $\mathrm{N}_{2}$ or $\mathrm{CO}_{2}$. As a rule replacing air by nitrogen intensifies the visible are spectrum (CN), but the ultra-violet below circa $3000 \AA$ becomes weaker as it loses most of its bands. 'The last-named advantage is partly compensated by the drawback that carbon electrodes in nitrogen disappear only very slowly so that in spectrochemical analysis a special technique may be necessary for accomplishing a sufficiently rapid vaporization of the sample. This difficulty does not come in when the air is replaced by $\mathrm{CO}_{2}$ instead of $\mathrm{N}_{2}$ to avoid the $\mathrm{NO}$ bands.

As appears from results of $P$. J. Somers the temperature measurement in the arc in nitrogen is hindered by the fact that the intensity profiles of the CN bands, which are often used for this purpose, are deformed by self-absorption.
\end{abstract}

BEI der Messung der Intensitäten von Emissionslinien eines zu untersurhenden Elementes muss der Untergrund des Bogenspektrums berücksichtigt werden. Der Einfluss dieses Untergrundes wächst mit dem Abnehmen der Linien-Intensität. Wird die Linie zu schwach, dann versinkt sie im Untergrund. Daher ist der Untergrund für die Messung von mittleren und kleinen Konzentrationen störend, währehd er bei sehr kleinen Konzentrationen die Messbarkeitsgrenze bestimmt.

Man kann zwar eine zu messende Spektrallinie mittels einer graphischen Methode für den Untergrund korrigieren [1], jedoch ist dies ziemlich zeitraubend und naturgemäss von begrenzter Genauigkeit. Besonders unangenehm wirkt die 'Tatsache, dass der Untergrund nicht gleichmässig ist, da er aus verschiedenen Molekülbanden besteht. Daher ist es wichtig, den Untergrund im Bogenspektrum zu vermindern; jedoch müssen hierbei die günstigen Eigenschaften des Bogens erhalten bleiben. Bei der Suche nach einer geeigneten Methode hierfür ist es 
zweckmässig, die Herkunft der verschiedenen Bestandteile des Untergrundes zu kennen.

Wir haben den Untergrund im ultravioletten Gebiet untersucht, und zwar von $3500 \AA$ bis $2300 \AA$. Nach der langwelligen Seite wird die Untersuchung durch dic starken CN-Banden begrenzt. In dem genannten Gebiet erwies sich die Struktur des Untergrundes beim Kohlebogen in Luft, ohne Analysensubstanz, als gut reproduzierbar. Auch die Intensität ist hinreichend konstant; Aufnahmen mit 3 Minuten Belichtungszeit stimmten bis auf ca. $5 \%$ überein. Hieraus kann mann

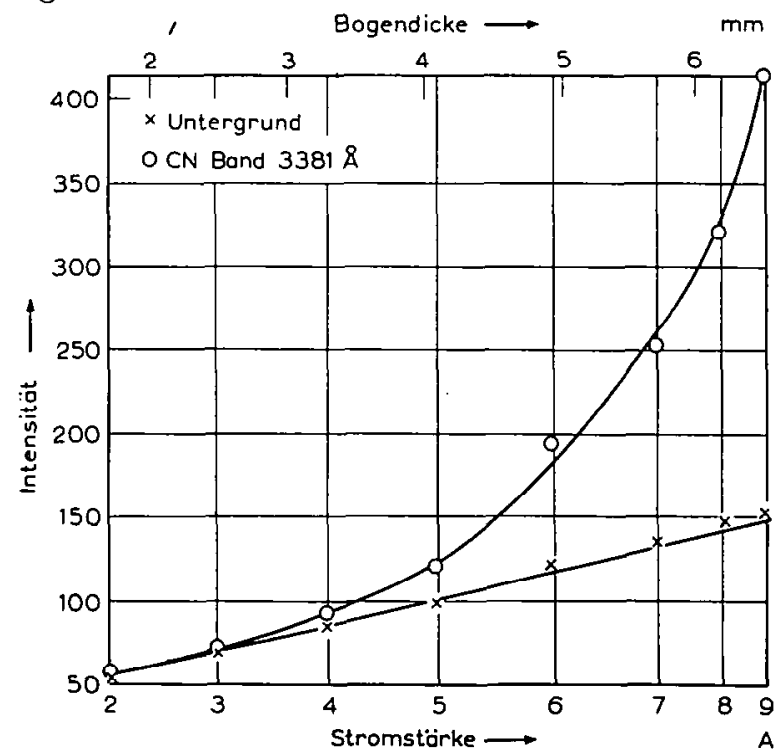

Abb. 1. Der Einfluss von Säulendicke (bzw.'Stromstärke) auf die Intensität der CN-Bande $3381 \AA$ und auf die des Untergrundes in der Umgebung dieser Bande.

schliessen, dass der Beitrag von glühenden Teilchen (z.B. Kohlepartikeln) am Untergrund des Bogenspektrums sehr gering ist. In der Tat erhielten wir mit harten und weichen Kohlestäben fast dasselbe Ergebnis. Eine nähere Untersuchung der Struktur des Untergrundes zeigt, dass der grösste Anteil dieses Spektrums aus zahlreichen Banden besteht. Dieses Bandenspektrum ist das Eigenspektrum des Kohlebogens in Luft; wir wollen aber bequemlichkeitshalber den Namen "Untergrund" beibehalten.

Im nahen Ultraviolett-nämlich zwischen $3500 \AA$ und $2900 \AA$-wurde eine Reihe sehr kurzer Banden gefunden, die anscheinend eine Forsetzung der violetten CN-Banden sind. Von diesen sogenannten "tail bands" ("Schwanz-Banden") [2] haben wir ungefähr zwanzig gefunden. Sie besitzen dieselbe starke Abhängigkeit von der Stromstärke im Bogen wie die bekannten violetten $\mathrm{CN}$-Banden. Die Zunahme der Bogen-Stromstärke hat auch eine Verstärkung des übrigen Untergrundes zur Folge. Diese Zunahme ist quantitativ aus der Zunahme der Bogensäulendicke zu erklären; die Emission pro Kubikzentimeter des Kernes bleibt ungefähr konstant. Die Intensität der CN-Banden nimmt aber stärker zu, als es durch die Dickenzunahme der Säule zu erklären ist (Abb. 1). Der Beitrag von den "tail bands" zum Untergrund ist nicht sehr wichtig, weil die Banden nur kurz sind; 
wohl wird der Untergrund dadurch weniger gleichmässig. In $\mathrm{N}_{2}$-Atmosphäre werden diese Banden stärker und in $\mathrm{CO}_{2}$ verschwinden sie, wie es auch zu erwarten ist.

Ein wesentlicherer Beitrag zum Untergrund im nahen Ultraviolett stammt vom Stickstoff und zwar von der zweiten positiven Gruppe. Die stärksten Banden dieses Systems. nämlich das Band bei $3371 \AA$ und $3159 \AA$ [3], sind deutlich wahrzunehmen (Abb. 2). Sie zeigen eine umfangreiche und intensive Rotationsstruktur.

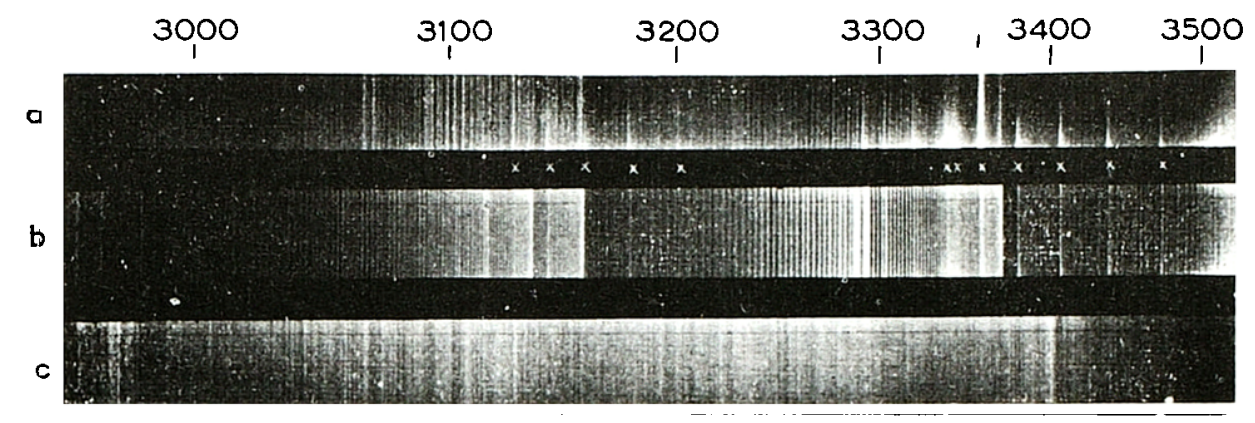

Abb. 2

(a) Eigenspektrum des Bogens in Luft. Fon 3400 bis ca. $3000 \AA$ sind $C X-$ und $\mathrm{N}_{2}$-Banden zu sehen.

Im Gebiet zwischen 3100 und $2800 \AA$ liegen $\mathrm{OH}$ - und andere Banden, die von sinuerstoff enthaltenden Molekiulen stammen.

(b) Eigenspektrum des Bogens in Stickstoff.

Die gekennzeichneten $(x)$ Banden stammen von $C X$, die anderen sind $x_{2}$-Banden.

(c) Eigenspektrum des Bogens in $\mathrm{CO}_{2}$.

Hier ist keine Utbereinstimmung mit den vorigen Aufnahmen \%u finden.

Das Streulicht im Spektrographen wurde gründlich unterdrückt durch Absorption des violetten $\mathrm{CN}$-Lichtes mit Hilfe eines Flüssigkeitsfilters, das mit $\mathrm{NiSO}_{4}-\mathrm{CoNO}_{4}-$ Lösung gefüllt war und sich vor dem Spalt befand.

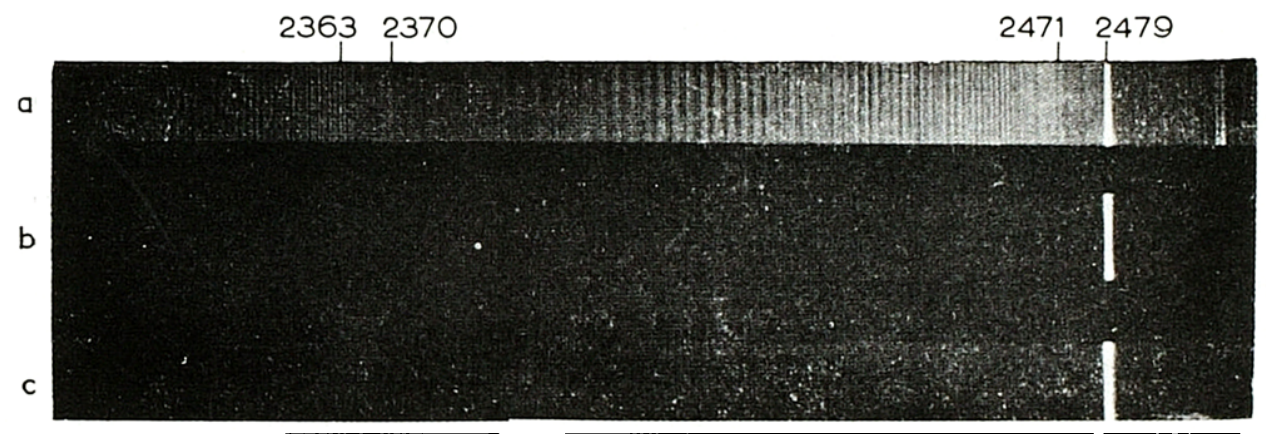

Abb. 3. (a) Eigenspektrum des Bugens in Luft, $\lambda=$ ca. 2500 bis ca. $2300 \mathrm{~A}$. Die Banden stammen vom NO-Molekül.

(b) Eigenspektrum des Bogens in Stickstoff. Die NO-Banden sind fast verschwunden.

(c) wie unter b), aber mit fünffacher Belichtungszeit.

Wenn der Bogen in einer Stickstoff-Atmosphäre brennt, kommen diese Banden und noch einige andere dieses Systems stärker zum Vorschein als in Luft. Dagegen sind sie, wie auch zu erwarten, in $\mathrm{CO}_{2}$-Umgebung nicht vorhanden.

Im ferneren Ultraviolett gibt es noch eine Reihe von Banden, die als das $\gamma$-System von NO identifiziert werden konnten. Die Banden $2722 \AA, 2680 \AA$, $2596 \AA$ und $2370 \AA$ [3] wurden mit Sicherheit festgestellt (Abb. 3). Die NO-Banden 
haben eine derart ausgedehnte Rotationsstruktur, dass der Untergrund beim Kohlebogen in Luft im Wellenlängengebiet von $2700 \AA$ bis $2300 \AA$ beinahe ausschliesslich aus diesen Banden besteht. Sie verschwinden vollkommen, wenn der Bogen in einer $\mathrm{N}_{2}$ - oder $\mathrm{CO}_{2}$-Umgebung brennt, das heisst, wenn entweder das $\mathrm{N}$ oder das O-Atom abwesend ist. Eine weitere Bestätigung, dass es sich hier um NO-Banden handelt, liefert die Tatsache, dass auch der Kupferbogen in Luft diese Banden aufweist.

Die erwähnten $\mathrm{CN}-, \mathrm{N}_{2^{-}}$und NO-Banden sind die markantesten Banden, die im Untergrund beim Bogen gefunden wurden. Sie besitzen alle einen ausgesprochenen Bandenkopf und (mit Ausnahme der kleinen $\mathrm{CN}-B a n d e n$ ) in regelmässigem Abstand angeordnete, gut identifizierbare Rotationslinien. Hierdurch ist aber nicht dér gesamte Untergrund erklärt. Ein grosser Teil dieses Spektrums des in Luft brennenden Bogens wird sichtbar überlappt durch ein oder mehrere Systeme von schwachen Linien, die in unregelmässigen Abständen angeordnet sind. Wegen des Fehlens von ausgeprägten Bandenköpfen, wegen der geringen Intensität und wegen der Anwesenheit der vorher genannten Banden ist es schwierig, um ohne besondere Massnahmen festzustellen von welchen Molekülen dieses Restspektrum herrührt.

Eine Untersuchung der Aussenzone des Bogens zeigt, dass in der Nähe von 3063 $\AA$ ein Teil dieser Linien durch die Aureole des Bogens ausgesendet wird. Das übrige Spektrum stammt ausschliesslich vom Kern des Bogens. Da wir vermuteten, dass diese Linien von einen $\mathrm{OH}-\mathrm{Band}$ herrühren, wurde der Bogen mit einer Gasflamme kombiniert. Dann ist nämlich reichlich Wasserstoff und Sauerstoff im Bogen vorhanden, sodass man starke $\mathrm{OH}-$ Banden erwarten kann. In der Tat kamen die gefundenen Banden nun stark zum Vorschein. Bei dem Bogen ohne Flamme versch winden diesc Banden fast vollständig, wenn trockene Luft aus einer Bombe verwendet wird. Dagegen weist der Kupferbogen in gewöhnlichem Luft diese $\mathrm{OH}-\mathrm{Banden}$ auf. Hieraus folgt, dass der benötigte Wasserstoff nicht von den verwendeten Kohlestäben herrührt. Der Wasserstoff stammt daher vermutlich vom Wasserdampf in der Luft.

Hiermit ist ein Teil des erwähnten, wenig strukturierten Spektrums erklärt. Der übrige Teil stammt vermutlich vom $\mathrm{O}_{2}$-Molekül. Brennt der Bogen in einer Stickstoff-Atmosphäre, dann ist dieser Teil nicht vorhanden. Dagegen zeigt der Kupferbogen in Luft dieses Spektrum. Hieraus folgt, dass das C-Atom nicht zu dem Molekül gehört, welches das Spektrum aussendet.

Dies alles zusammenfassend, können wir feststellen, dass der Untergrund des Bogens in Luftatmosphäre im Wellenlängengebiet von 3500 bis $2300 \AA$ hauptsächlich aus $\mathrm{N}_{2^{-}}$, NO- und vermutlich $\mathrm{O}_{2}$-Banden besteht. Ferner finden wir dort noch einen wenig störenden Untergrund der von kurzen CN-"tail bands" abstammt, während ein $\mathrm{OH}$-Band nur auftritt wenn die Luft feucht ist. Der Untergrund wird also von drei wesentlichen Elementen hervorgerufen: $\mathrm{C}, \mathrm{O}$, und $\mathrm{N}$.

Arbeiten wir mit einem Kohlebogen, dann wird sich naturgemäss immer Kohlenstoff im Bogen befinden. Durch Verwendung eines Bogens, der in $\mathrm{CO}_{2}$ oder $\mathrm{N}_{\mathrm{2}}$-Umgebung brennt, kann die Anzahl der möglichen Banden verringert werden. Benutzt man eine $\mathrm{CO}_{2}$-Atmosphäre, dann treten jedoch eine grössere Anzahl anderer Banden auf, wodurch der Untergrund ungefähr genau so intensiv 
ist wie in Luft, jedoch ist er im nahen Ultraviolett gleichmässiger (Abb. 2). Dies bringt also keinen grossen Vorteil. Die Verwendung von Stickstoff lässt die bei der Luftatmosphäre auftretenden $\mathrm{CN}$ - und $\mathrm{N}_{2}$-Banden stärker zum Vorschein kommen. Ausserdem tritt das $\mathrm{N}_{2}$-Band $2979 \AA$ auf (Abb. 2). Dies hat zur Folge, dass das Gebiet zwischen $3500 \AA$ und $2900 \AA$ einen intensiven Untergrund mit viel Struktur besitzt. Bei Wellenlängen kleiner als $2900 \AA$ ist die Sachlage aber ganz anders. Hier sind alle Banden fast vollständig verschwunden (Abb. 3). Die Intensität dieses Spektrums beträgt hier noch ungefähr 5 bis $10 \%$. verglichen mit dem

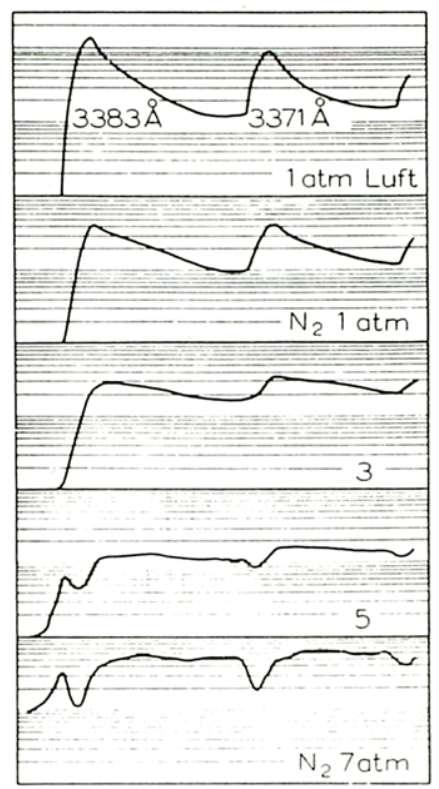

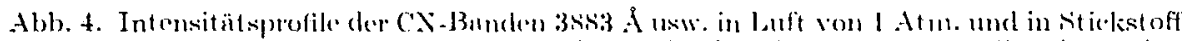

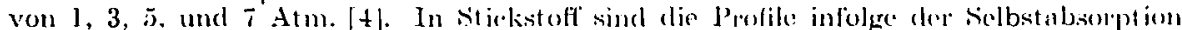

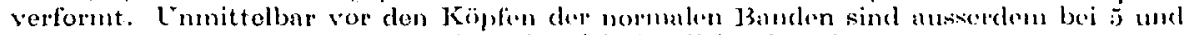
7 Atm. ('X."tail bands" deutlich crkemubar.

Sipektrum bei Luftatmosphäre. Hauptsärchlich besteht dieses Spelitrum aus sebr schwachen Resten von Banden, die auch bei Luftatmosphäre gefunden werden. Wahrscheinlich entstehen sie, weil der verwendete Stickstoff noch Spuren von Sauerstoff als Verunreinigung enthält. Es ist schwierig festzustellen. ob es hier auch ein echtes Kontinum gibt; es kann dam jeclenfalls nur sehr schwarh sein.

Der Untergrund im Gebiet von $2900 \AA$ bis $2300 \AA$ ist also beim Bogen in Stickstoff-Atmosphäre sehr viel schwicher als beim in Luft bremnenden Bogren. Ausserdem bremt dieser l3ogen sehr ruhig, sodass auf diese $\mathrm{W}^{\dagger}$ eise eine in cliesem Wellenlängengebiet geeignete Lichtquelle für z.B. spektrochemische ['nfersucllungen gefunden zu sein scheint. Ein Nachteil ist aber mit diesem Bogen verbunden: Das Abbrennen der Kohlestäbe des Bogens in Luft-Atmosphäre entsteht durch das Verdampfen und Verbrennen des Kohlenstoffs. Die Abbrenngeschwindigkeit, die hauptsächlich dic Mcnge der pro Sckunde in den Bogen gelangenden Analysensubstanz bestimmt, ist ziemlich gross. Beim Bogen in Stickstoff-Atmosphäre wird das "Abbrennen" nur clurch das Verdampfen der Kolılestäbe verursachlt. Die 
Geschwindigkeit hiervon ist sehr gering, sodass die Analysensubstanz nur langsam in den Bogen gelangt. Um diesen Nachteil zu beseitigen, würde eine spezielle Form der gefüllten Elektrode notwendig sein.

Zum Abschluss noch eine kurze. Bemerkung über den Bogen in StickstoffAtmosphäre: Nach den Ergebnissen von P. J. Sovers [4] wird die Temperaturmessung im Bogen in Stickstoff-Atmosphäre dadurch erschwert, dass die gebräuchlichen CN-Banden Selbstabsorption zeigen, wodurch ihre Intensitätsprofile verformt werden (Abb. 4).

\section{Literatur}

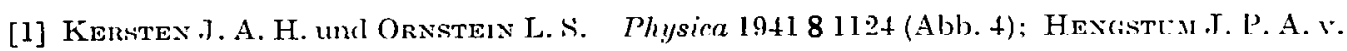
uncl S.ut .J. A. Physica $195622 \times 6$ (Abb. 3).

[2] Feast M. W. I'roc. Phys. SHoc. 194922121.

[3] Pearse R. W. B. und Gaymon A. (r. The inlentification of molecular spectra London, 1950.

[4]. Somers P. J. und Simit J. A. Appl.sici. Res. 1956 B6 1.

\section{Diskussion}

H. Rüssmann: Haben sie Unterschiede in clen sipelitren der verschiedenen Kohlearten festgestellt? Besteht ein Unterschied in der Art des Untergrundes bei Kohle- oder (iraphitelektroclen?

Antwort: Nein; nein.

H. HaFTkA: Wir haben Unterschiede bei den Sipoktren von graphitischen und nichtgraphitischen Kohlen festgestellt. Vor allen Dingen zeigten die CII Linien bei $2830 \AA$ und $2500 \AA$ eine stärkere Intensität bei den nicht-graphitischen Kohlen.

Antwort: Es hat keine messbare Anderung der Untergrundintensität zufolge.

H. HaftKa: Wir möchten sagen, dass die durch die verschiedene Würmeleitfähigkeit bedingte unterschiedliche Temperatur die Ursache des verstörten Auftretens der (' II Linien bei den wesentlich heisseren nicht-graphitischen Kohlen ist.

H. Lehmann: Die Unterschiede im Abbrand der Kohleelektroden in Luft und stickstoff lassen sich ausgleichen, wenn man im Stickstoff hochgraphiticrto Elcktroden verwendet, dic einèn ausreichend starken und gleichmässigen Abbrand in Stickstoffatmosphäre zeigen.

H. vaN CALKER: "Nicht graphitierte" Elektroden haben ein anderes Wärmeleitvermögen; dann ist das Produkt von Brennfleckdurchmesser und Temperatur geändert und es ist zu verstehen, dass bei der höheren 'Temperatur die C II Linie stärker erscheint.

H. Addink: Es könnte möglich sein den Abbrand in $\mathrm{N}_{2}$ zu kompensieren miltels einer zusätzlichen Erhitzung der Anode von aussen.

Antwort: Das haben wir noch nicht ausprobiert.

H. Holdt: Sind Versuche gemacht bei fehlendem Kohleabbrand (keine O-hältige Atmosphäre) die sehr starke Anfangsverdampfung der ersten Sekunden für die quantitative Analyse zu verwenden um den mangelnden Abbrand zu kompensieren?

Antwort: Keine Versuche.

H. Russmann: Wie verändert sich der Untergrund bei Pufferung mit Alkali?

Antwort: Die CN-Banden, die $\mathrm{N}_{2}$-Banden und die NO-Banden verschwinden fast völlig bei dieser niedrigen Temperatur des Bogens. Die $\mathrm{OH}$-Banden und die vermuteten $\mathrm{O}_{2}$-Banden zeigen fast die selbe Intensität.

H. van Calker: Haben Sie Banden des aktiven Stickstoffs im Gelben beobachtet?

Antwort: Nur das U.V. ist von mir untersucht worden. 